\title{
Numerical Simulations of Discharge Change Propagation in Open Channel Irrigation Systems
}

KHANH, Mai Quoc

Laboratory of Water Environment Engineering, Course of Bioproduction Environmental Sciences, Department of Agro-Environmental Sciences, Graduate School of Bioresource and Bioenvironmental Sciences, Kyushu University

HIRAMATSU, Kazuaki

Laboratory of Water Environment Engineering, Course of Bioproduction Environmental Sciences, Department of Agro-Environmental Sciences, Graduate School of Bioresource and Bioenvironmental Sciences, Kyushu University

TABATA, Toshinori

Laboratory of Water Environment Engineering, Course of Bioproduction Environmental Sciences, Department of Agro-Environmental Sciences, Graduate School of Bioresource and Bioenvironmental Sciences, Kyushu University

HARADA, Masayoshi

Laboratory of Agricultural Machinery and Production Systems Design, Division of Bioproduction Environmental Sciences, Department of Agro-environmental Sciences, Faculty of Agriculture, Kyushu University

https://doi.org/10.5109/2558906

出版情報 : 九州大学大学院農学研究院紀要. 65 (1)，pp.139-148，2020-02. Faculty of Agriculture， Kyushu University

バージョン :

権利関係 : 


\title{
Numerical Simulations of Discharge Change Propagation in Open Channel Irrigation Systems
}

\author{
Mai Quoc KHANH ${ }^{1,2}$, Kazuaki HIRAMATSU, Toshinori TABATA ${ }^{3}$ \\ and Masayoshi HARADA
}

\author{
Laboratory of Water Environment Engineering, Division of Bioproduction Environmental Sciences, \\ Department of Agro-Environmental Sciences, Faculty of Agriculture, \\ Kyushu University, 744 Motooka, Nishi-ku, Fukuoka 819-0395 \\ (Received October 31, 2019 and accepted November 14, 2019)
}

\begin{abstract}
The purpose of this study is to investigate the arrival times of discharge change propagation from upstream to downstream for improvement of irrigation efficiency. The arrival time of discharge change propagation was defined as the time required at a selected section of a canal system under steady flow conditions with a discharge $Q$ to change to steady flow conditions with a discharge of $Q \pm \Delta Q$ when the upstream end discharge is increased or decreased by $\pm \Delta Q$. A one-dimensional gradually varied flow model was constructed and numerically calculated using an explicit finite difference method of the Leapfrog scheme in an artificial irrigation canal of Horikawa-Yousui Irrigation Canal, Japan, and a natural river of Kim Son River, Vietnam. The simulations for validation and scenario analysis were conducted in irrigation periods of 2018 and 2019 for both the canal and river, respectively. In the validation, root mean square errors (RMSE) were used to evaluate the simulation performance, and the calculated RMSEs indicated a good result considering the slight errors between the simulated and observed data. In the scenario analysis, the observed maximum and minimum discharges in both the canal and river in the irrigation periods were utilized in setting scenarios, and the simulations were conducted considering diversion discharges and no diversion discharges. On average, the results of scenario analysis indicated that the arrival time of discharge change propagation from upstream to downstream for the Horikawa-Yousui Irrigation Canal was approximately $1-1.5 \mathrm{~h}$ for a length of $4.5 \mathrm{~km}$. However, the discharge change propagation was approximately 10 to $20 \mathrm{~h}$ for the Kim Son River that had a length of $14.2 \mathrm{~km}$ to achieve a nearly steady state at the downstream end. The most important finding was that higher discharge increasing or decreasing normally led to higher arrival times of discharge change propagation from upstream to downstream. Besides, a comparison between the celerity of surge propagation and the average velocity of discharge change propagation implied that the effects on these propagations due to longer or shorter time of opening gate led to a gradual or sudden discharge change propagation. So far, the one-dimensional gradually varied flow model with its advantages could be applied for a network system with nodes or a cycling route of channels.
\end{abstract}

Key words: one-dimensional gradually varied flow, Leapfrog scheme, scenario analysis, arrival time, surge propagation

\section{INTRODUCTION}

An increasing world population, as a main humanitarian problem, has become a matter of serious concern in the $21^{\text {st }}$ century in terms of climate change and sustainable development. Subsequently, to meet the human's demands on food consumptions in 2050, global crop production should be twice compared with that in the past (Jägermeyr et al., 2015). Among the many solutions proposed to achieve that goal, improving irrigation efficiency is an important factor to maintain optimal crop productivity rates in a sustainable manner by minimizing water because of the scarcity of water that will become a serious danger for human in the future. Obviously, farmers could not cultivate their crops without irrigation and plants could not be able to survive without water. There has been evidence that food secu-

\footnotetext{
1 Laboratory of Water Environment Engineering, Course of Bioproduction Environmental Sciences, Department of AgroEnvironmental Sciences, Graduate School of Bioresource and Bioenvironmental Sciences, Kyushu University

2 Center for Participatory Irrigation Management, 171 Tay Son, Dong Da, Hanoi, Vietnam

Corresponding author (E-mail: ttabata@bpes.kyushu-u.ac.jp)
}

rity has increased and living standards have improved through irrigation in many corners of the world (Meinzen-dick, 2014). Irrigation serving agriculture has been focused on by not only governments but also farmers, especially in developing countries belonging to Asia or Africa regions.

Recently, with the rapid development of technology, farmers have paid more attentions on the development of irrigation systems. At present, irrigation is not merely controlled and monitored manually. In fact, it is being modernized and automated. The application of technological advances in irrigation systems would save water. One of the common technological advances is called information communication technology (ICT). As can be clearly seen, ICT is very useful for agriculture, especially in terms of improving water use efficiency. Many irrigation systems have been introducing ICT to successfully save water during irrigation. Hence, the evaluation of operations in the abovementioned irrigation system assisted with the ICT is indispensable. Knowing time delays or the arrival times of discharge change propagation are key tools for such evaluation.

Saint-Venant equations are normally applied to express one-dimensional problems in irrigation channels 
or natural rivers (Ferreira et al., 2017). The SaintVenant equations (can be called hydrodynamic model), which include expressions of mass and momentum conservation, concisely describe the gradually varied flow in an open channel (Chaudhry, 2008) through spatial and temporal representation of longitudinal flow closer to reality. With the purpose of solving the Saint-Venant equations, numerical schemes, including methods of characteristics, finite difference method, and finite element method, have been successfully applied to a hydraulic model (Zarmehi et al., 2011).

In relation to approaches for constructing a onedimensional flow model in an open channel, many research studies have been successfully conducted, in which Chaffranek (1987) developed a one-dimensional flow model for simulating unsteady flow in a single open-channel reach or in a network of interconnected channels by using the four-point box scheme to solve the Saint-Venant equations. However, in this study, the author mentioned the effects of wind in the governing equations, that may cause some differences in results. Ferreira et al. (2017) constructed a one-dimensional flow by using the Lax diffusive scheme (explicit finite difference scheme) to solve the Saint-Venant equations and comparing the results with the HEC-RAS model. As a result, the diffusive Lax method was shown to be significantly more restrictive and sensitive to changes in the interval of time discretization. Horvat et al. (2017) and Fang et al. (2008) also used the same numerical scheme, i.e., Preissmann's implicit finite difference method; however, the shortcomings of this approach were the difficulty in calibration itself and poor results for discharges that alter from the ones used for calibration.
In this research, the arrival times of discharge change propagation were numerically and quantitatively investigated so that the output results can be used as a database and play an important role in terms of the automatic operation for irrigation systems. The arrival time of discharge change propagation was defined as the time required at a selected section of a canal system under steady flow conditions with a discharge $Q$ to change to steady flow conditions with a discharge of $Q \pm \Delta Q$ when the upstream end discharge is increased or decreased by $\pm \Delta Q$. A one-dimensional gradually varied flow model was developed and numerically solved using the Leapfrog numerical scheme because of its simplicity and low computational time. Subsequently, the optimal discharge change propagation considering various discharge levels were proposed based on the simulation results.

\section{MATERIALS AND METHODS}

\section{Targeted canal and river}

\section{Horikawa-Yousui Irrigation Canal}

The first study area, i.e., the Horikawa-Yousui Irrigation Canal located in Asakura City, Fukuoka Prefecture, Japan, is shown in Fig. 1. It is an irrigation canal system that receives water from the Chikugo River through the Yamadazeki Barrage. The targeted canal has a length of $4.6 \mathrm{~km}$ along the main line, which starts from the gate monitor at the upstream end of the HorikawaYousui Irrigation Canal. The average bottom gradient of the targeted canal is 0.0005 . To solve the hydrodynamic model and conduct scenario analyses for the arrival time of discharge change propagation using the model, the following data were required: cross-sectional and longi-

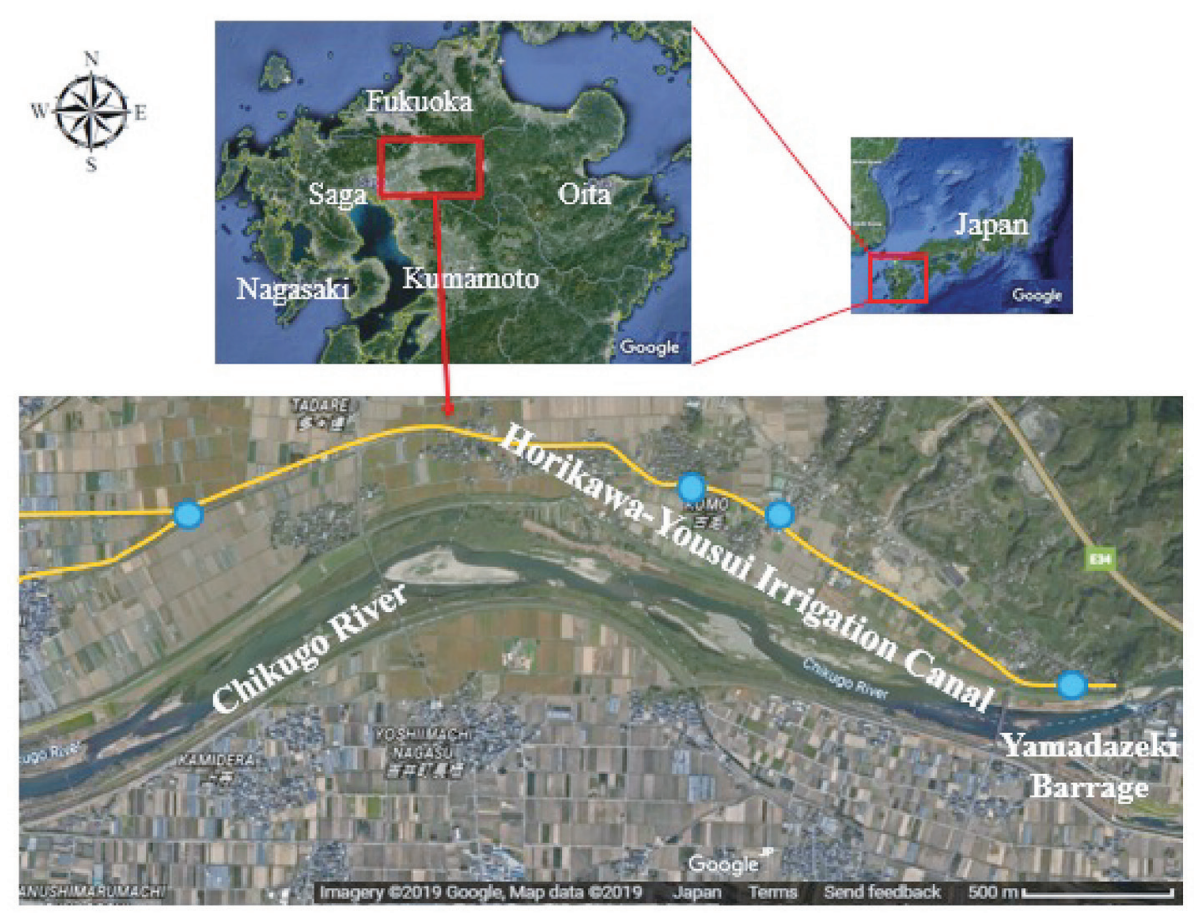

Fig. 1. Location of the Horikawa-Yousui Irrigation Canal. The blue circles indicate the observation points of water depth and flow velocity. 
tudinal profiles of the canal, diversion discharges and maximum and minimum discharges, and water levels during the irrigation period. In addition, the data series of observed discharge at the upstream end and observed water level at the downstream end were collected to determine the boundary conditions.

\section{Kim Son River}

The second study area, i.e., the Kim Son River with a length of approximately $61.7 \mathrm{~km}$, is shown in Fig. 2. It is one of the four main rivers located inside the Bac Hung Hai Irrigation System, and it supplies water to the entire system through the Xuan Quan sluice gate at the upstream end connecting the Kim Son River and Red River. The targeted section for simulation is the upstream end of the Kim Son River, and it has a length of $14.2 \mathrm{~km}$, starting from the Xuan Quan sluice gate and ending at the Kenh Cau sluice gate. The average bottom gradient of the targeted river is 0.000014 , which means nearly horizontal bottom gradient. Between these two sluice gates, there is another sluice gate called the Bao Dap, which is an important validation position in this study. There are four gates with a width of $4.0 \mathrm{~m}$ and height of $3.5 \mathrm{~m}$, and one navigation gate at the Xuan Quan sluice gate, which is the same as that of the Bao Dap sluice gate. Each gate has a width of $5.0 \mathrm{~m}$ and height of $4.15 \mathrm{~m}$. The Kenh Cau sluice gate has six gates and one navigation gate, and each gate has a width of $3.2 \mathrm{~m}$ and height of $3.7 \mathrm{~m}$.

Similar to the hydrodynamic model in the HorikawaYousui Irrigation Canal, the data required for the Kim Son River included the cross-sectional and longitudinal profiles, diversion discharges and data series of observed water level at the upstream end (Xuan Quan sluice gate), the downstream end (Kenh Cau sluice gate), and the validation position (Bap Dap sluice gate). The water level was observed at the upstream and downstream sides of each sluice gate. With respect to the boundary condition at the upstream end, discharge was calculated by the observed water levels at the upstream and downstream sides of the Xuan Quan sluice gate and discharge formulas for submerged flow and complete flow.

\section{Governing equations and numerical scheme}

A one-dimensional gradually varied flow in open channels can be described by two partial-differential equations expressing mass and momentum conservation (Schaffranek, 1987), called the Saint-Venant equations, which are represented as follows:

- Continuity equation using to calculate water level:

$$
B \frac{\partial H}{\partial t}+\frac{\partial Q}{\partial x}=q
$$

- Momentum equation using to calculate discharge:

$$
\begin{aligned}
\frac{\partial Q}{\partial t}+\frac{2 Q}{g A} \frac{\partial Q}{\partial x}+ & \left(1-\frac{Q^{2} B}{g A^{3}}\right) \frac{\partial H}{\partial x} \\
& +\frac{n^{2} Q|Q|}{A^{2} R^{4 / 3}}=\frac{q w^{\prime} \cos \theta}{g A}
\end{aligned}
$$

where $x$ is the longitudinal distance along the canal or river bottom from the upstream end $(\mathrm{m}), t$ is the time (s), $B$ is the surface width (m), $H$ is the water level (m), $Q$ is the discharge $\left(\mathrm{m}^{3} / \mathrm{s}\right), q$ is the lateral contribution per unit of channel length $\left(\mathrm{m}^{3} / \mathrm{s}\right), A$ is the cross-sectional area $\left(\mathrm{m}^{2}\right), g$ is the acceleration of gravity $\left(\mathrm{m} / \mathrm{s}^{2}\right), n$ is the Manning's roughness coefficient $\left(\mathrm{s} / \mathrm{m}^{-1 / 3}\right), R$ is the hydraulic radius (m), $w$ is the velocity of side flow $(\mathrm{m} / \mathrm{s})$, and $\theta$ is the angle between lateral contribution and main flow (rad).

Numerical techniques are used for obtaining the solutions to the Saint-Venant equations: (i) a method of characteristics, (ii) finite difference methods, and (iii)

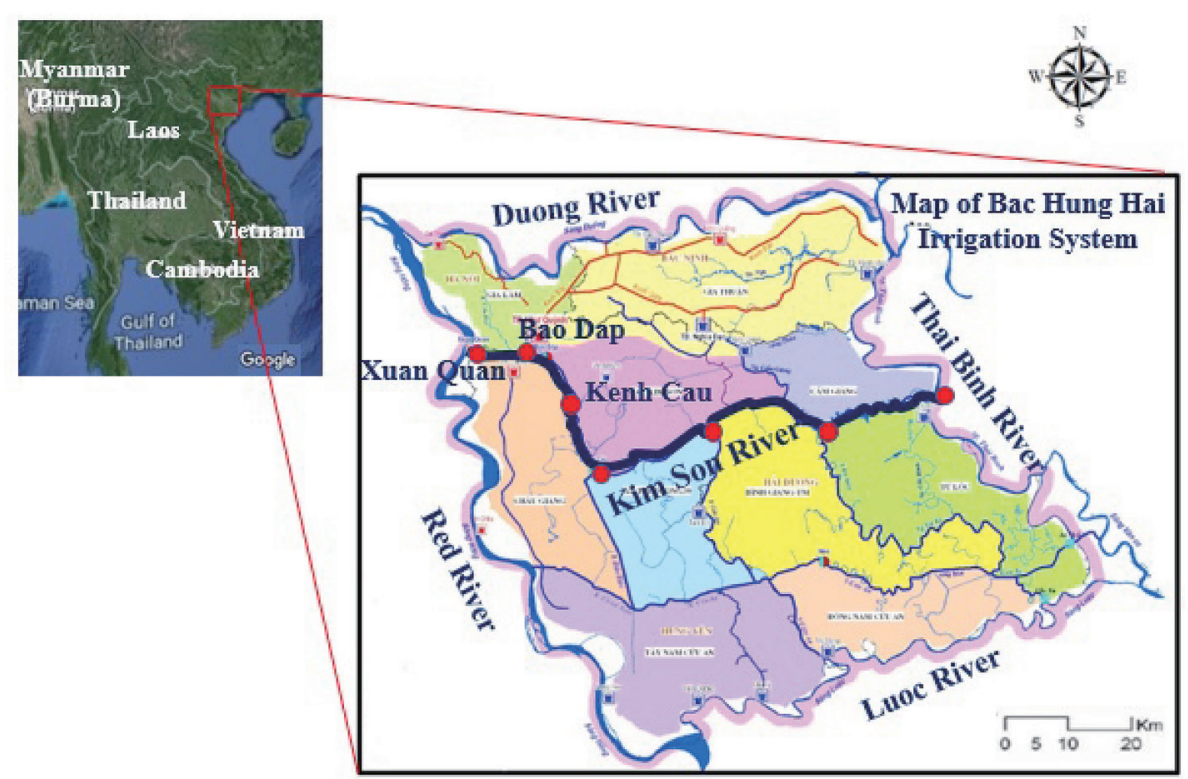

Fig. 2. Location of the Kim Son River. The red circles show the sluice gates along the Kim Son River. 
finite element methods.

Of these methods, the finite element method is rarely used when flow is approximated as one-dimensional flow such as in the case of Saint-Venant equations. The other two methods have been commonly applied to obtain the numerical solution to a one-dimensional unsteady flow since 1960s. The finite difference methods can further be classified as explicit and implicit techniques, each of which holds distinct numerical characteristics. For example, some usual explicit schemes include: Foward-Time-Centered-Space, MacCormack, QUICK, Leapfrog and Lax Wendroff. While, some common implicit representations usually are BackwardTime-Centered-Space, Crank-Nicolson and Preissman. Explicit methods are less complex for implementation, but its stability is conditioned to the simulation time interval. In implicit methods, the definition of temporal discretization is less restrictive than that of explicit methods, although some studies have indicated loss of quality in the results when increasing the Courant number or even inefficient in terms of the use of computational time ( Ferreira et al., 2017).

Considering the abovementioned advantages of numerical techniques, the explicit finite difference technique is commonly used to solve the governing partial differential equations. In this study, an explicit finite difference method, i.e., the Leapfrog scheme was used to solve the Saint-Venant equations, and FORTRAN 90 was used as the complied programming language that can optimize the simulation time. The Leapfrog method takes the central difference both in time and space, alternately obtains the water level $H$ and flow rate $Q$. For this reason, this scheme is very convenient and simplified to implement. The stability calculation of the Leapfrog scheme is based on the Courant-FriedrichsLewy condition, which is given as follows:

$$
\Delta t \leq \frac{\Delta x}{\max |V \pm \sqrt{g h}|}
$$

where $\Delta t$ is the interval of time discretization (s), $\Delta x$ is the interval of spatial discretization $(\mathrm{m}), V$ is the maximum velocity $(\mathrm{m} / \mathrm{s})$, and $h$ is the water depth $(\mathrm{m})$.

\section{Boundary conditions and calculation conditions}

The boundary conditions in the Horikawa-Yousui Irrigation Canal and Kim Son River are listed in Table 1, including those in the upstream (discharge) and downstream ends (water level). The boundary conditions in validation included the observed discharge at the upstream end and observed water level at the downstream end. The calculated water level at the downstream end was also utilized by introducing the uniform flow assumption, which was based on Manning's equations (Chow, 1959; Akgiray, 2005). In the uniform flow assumption, the water level was calculated through repeated calculation based on Manning's equation and the discharge at a previous time step at a neighboring calculation point. In the scenario analyses, for the arrival time of discharge change propagation, the uniform flow assumption was applied to calculate the water level at the downstream end.

Especially, for the case of Kim Son River, the upstream discharge boundary condition was calculated by the submerged overflow and complete overflow equations using the observed data series of water level at the upstream and downstream ends of the Xuan Quan sluice gate.

Because the Horikawa-Yousui Irrigation Canal is a concrete canal, the Manning's coefficient was chosen as $0.025 \mathrm{~s} / \mathrm{m}^{1 / 3}$. As the Kim Son River is a natural river, the Manning's coefficient was chosen as $0.035 \mathrm{~s} / \mathrm{m}^{1 / 3}$ (Chow, 1959). Besides, the chosen spatial distance $\Delta x$ was $100 \mathrm{~m}$ and $200 \mathrm{~m}$ for the case of the Horikawa-Yousui Irrigation Canal and Kim Son River, respectively, while the time difference $\Delta t$ for calculation was $1 \mathrm{~s}$ for both these cases.

\section{Moving average technique}

With respect to the Kim Son River, it was necessary to apply the moving average technique for smoothing the bottom elevation, as shown in Fig. 3. The reason behind this was that there were a few heavy steep slopes of the bottom elevation in the Kim Son River in which the bottom elevation suddenly increased or decreased, as can be seen in that figure. By applying the moving average, the calculation of water level and discharge became stable and smooth, and good results were obtained successfully.

\section{RESULTS AND DISCUSSION}

\section{Validation of developed models}

To validate the model, two validation options were conducted. The first validation used the observed discharge at the upstream end and water level at the downstream end as boundary conditions. Another validation used the observed discharge at the upstream end and calculated water level via the uniform flow assumption

Table 1. Boundary conditions used in the Horikawa-Yousui Irrigation Canal and Kim Son River

\begin{tabular}{|c|c|c|c|c|}
\hline \multicolumn{2}{|c|}{ Targeted canal/river } & \multirow{2}{*}{$\begin{array}{c}\text { Upstream boundary } \\
\text { conditions }\end{array}$} & \multicolumn{2}{|c|}{ Downstream boundary conditions } \\
\hline $\begin{array}{l}\text { Horikawa- } \\
\text { Yousui } \\
\text { Irrigation } \\
\text { Canal/ }\end{array}$ & Validation & & $\begin{array}{l}\text { Observed water } \\
\text { level }\end{array}$ & $\begin{array}{l}\text { Calculated water level } \\
\text { using uniform flow } \\
\text { assumption }\end{array}$ \\
\hline $\begin{array}{l}\text { Kim Son } \\
\text { River }\end{array}$ & $\begin{array}{l}\text { Scenarios } \\
\text { analyses }\end{array}$ & Varying discharge & \multicolumn{2}{|c|}{$\begin{array}{l}\text { Calculated water level using uniform flow } \\
\text { assumption }\end{array}$} \\
\hline
\end{tabular}




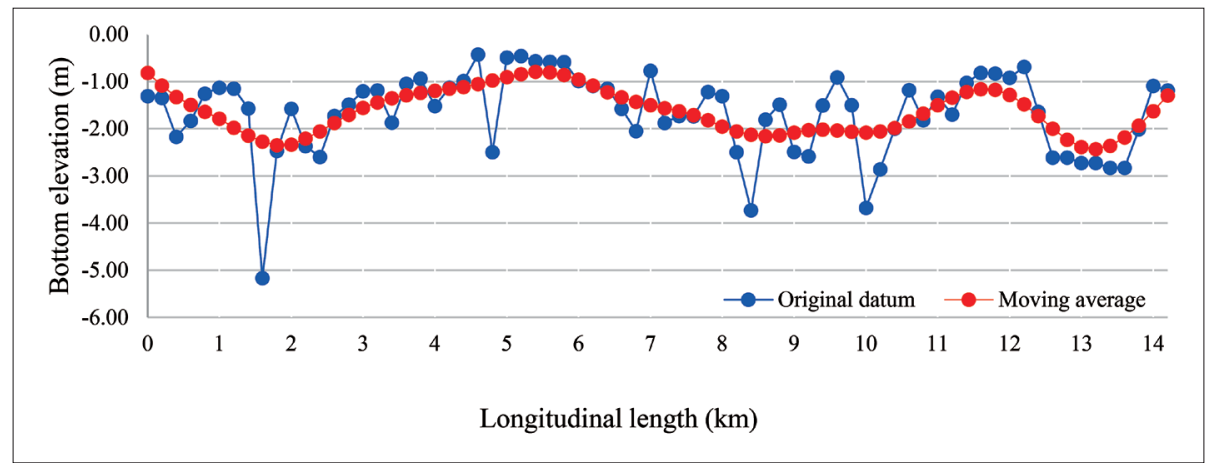

Fig. 3. Five-point moving average of bottom elevation for the Kim Son River.

for calculating the water level at the downstream end as boundary conditions. For detail, in the case of Horikawa-Yousui Irrigation Canal, the validations were performed using observed water levels and flow velocities at four positions: one near the upstream end, two at the middle of canal, and one at the downstream end, as shown in Fig.1. The observation was accomplished once in September $8^{\text {th }}, 2018$ during the irrigation period. In regard to the Kim Son River, the validation was performed by using the data series of the observed water level at the Bao Dap sluice gate obtained four times per day at 01:00, 07:00, 13:00, and 19:00 during the irrigation

(1) Discharge

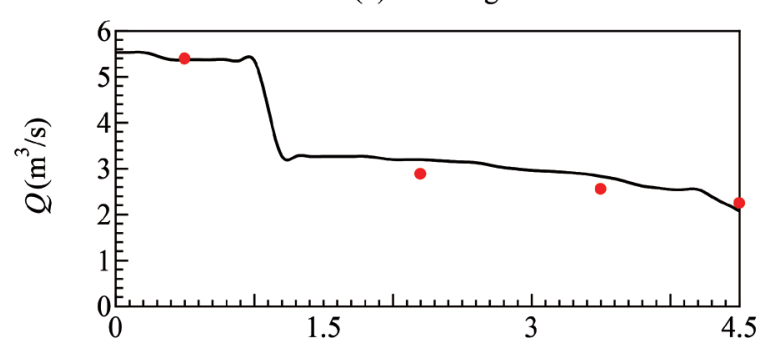

(2) Flow velocity

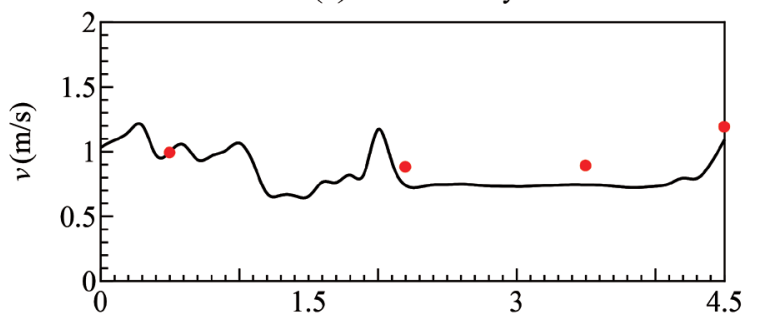

(3) Water level

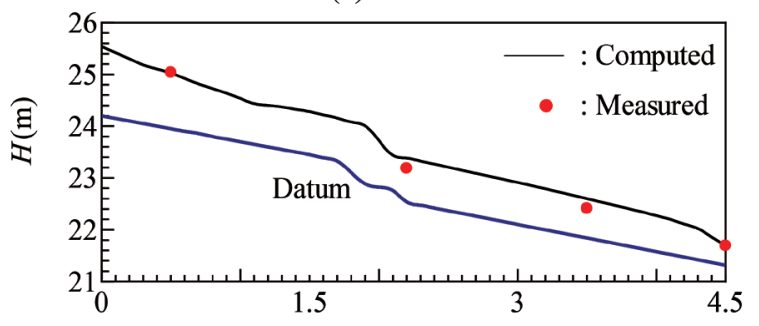

Longitudinal length from upstream end in $x$ - direction $(\mathrm{km})$

Fig. 4. Validation of the model using the observed discharge and water level in the Horikawa-Yousui Irrigation Canal. period starting from January $22^{\text {nd }}, 2019$, to January $24^{\text {th }}$, 2019.

Figures 4 and 5 showed the model validation results for the Horikawa-Yousui Irrigation Canal using (i) the observed discharge at the upstream end and the observed water level at the downstream end as boundary conditions, and (ii) the observed discharge at the upstream end and the calculated water level at the downstream end via uniform flow assumption, respectively. Figures 6 and 7 indicated the validation for the Kim Son River in regard to same cases as the HorikawaYousui Irrigation Canal. As can be seen from the figures,

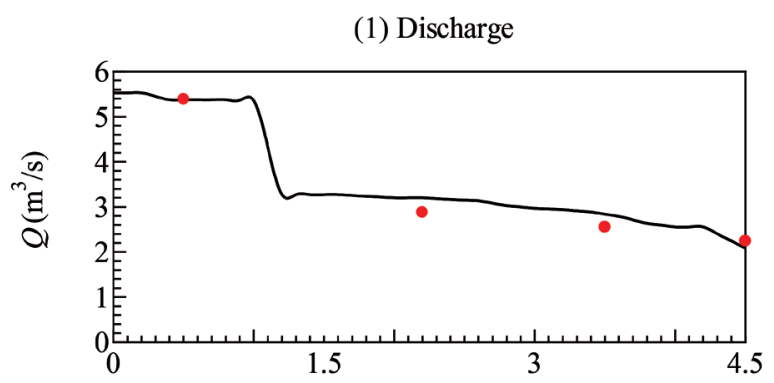

(2) Flow velocity

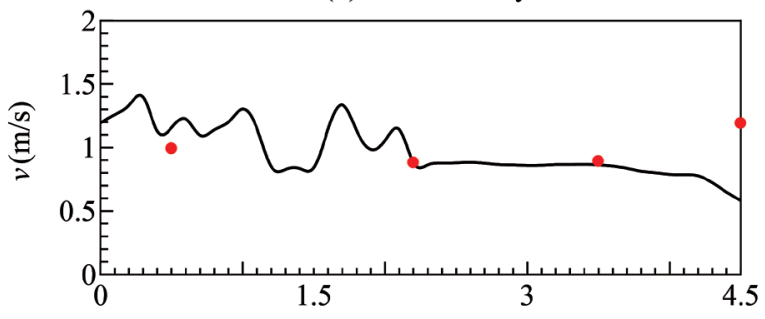

(3) Water level

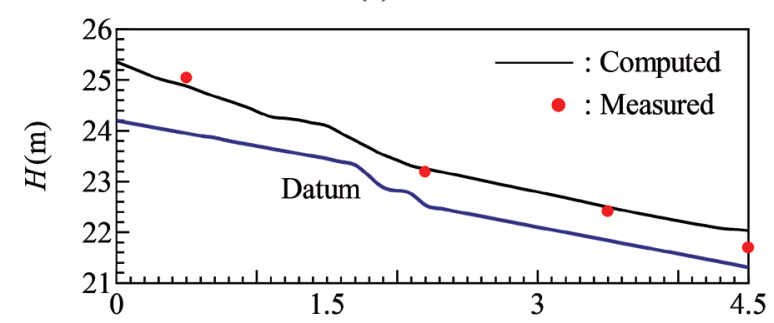

Longitudinal length from upstream end in $x$ - direction $(\mathrm{km})$

Fig. 5. Validation of the model using the observed discharge and uniform flow assumption in the Horikawa-Yousui Irrigation Canal. 
using observed discharge at the upstream end and the observed water level at the downstream end as boundary conditions, the simulation results more precisely matched the observed data than the case of using uniform flow assumption. Tables 2 and 3 showed the rootmean-square error (RMSE) indicator for the validations using (i) the observed discharge at the upstream end and the observed water level at the downstream end as boundary conditions and (ii) the observed discharge at the upstream end and the calculated water level at the downstream end via uniform flow assumption for both the Horikawa-Yousui Irrigation Canal and the Kim Son River. It was concluded that in both cases, the RMSEs were in the allowable range, and the simulation results demonstaretd good performance compared to the observed ones.

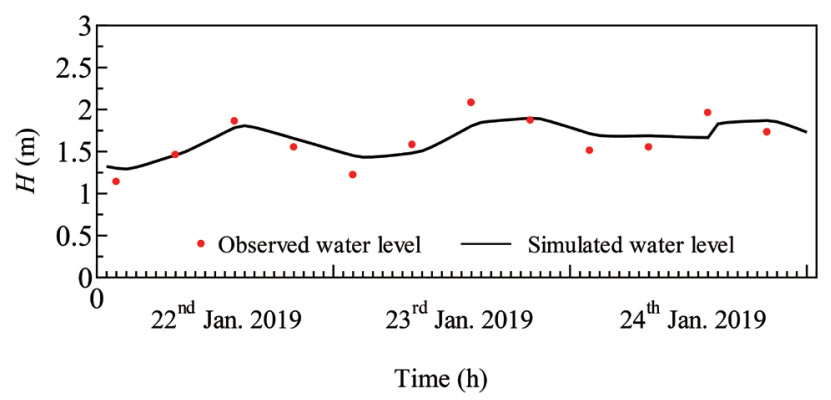

Fig. 6. Validation of the model using the observed discharge and water level in the Kim Son River.

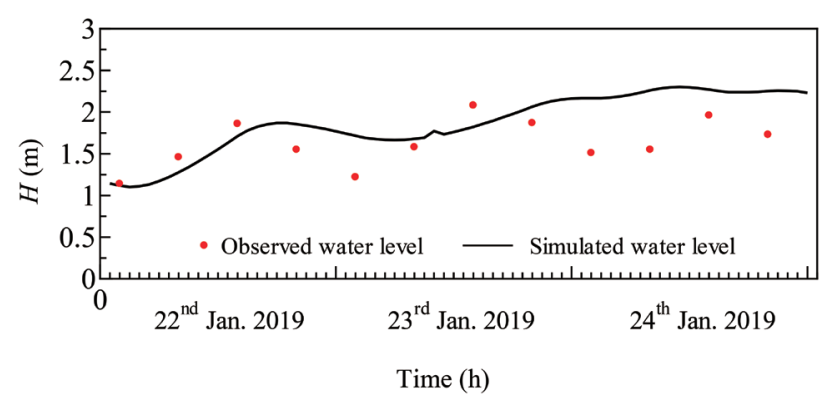

Fig. 7. Validation of the model using the observed discharge and uniform flow assumption in the Kim Son River.

\section{Scenarios analyses of arrival time}

The arrival time of discharge propagation is determined as the time required at a selected section for a canal system under steady flow conditions with a discharge $Q$ to change to steady flow conditions with a discharge of $Q \pm \Delta Q$ when the upstream end discharge is increased or decreased by $\pm \Delta Q$ (Kitamura, 1990). In this study, the arrival time or time delay was understood as having the same meaning as travel time/flow-arrival time of discharge change; however, for more clarity, it was defined as the time required to reach to the discharge which equals to $95 \%$ for the case of increasing discharge or $105 \%$ for the case of decreasing discharge of the targeted discharge under the nearly steady stage at the downstream end.

In the case of the Horikawa-Yousui Irrigation Canal, the scenarios of arrival time of discharge change propagation were set-up considering no diversion discharge. There were five levels of discharge change determined from the maximum and minimum discharge during the irrigation period. The minimum discharge during irrigation period was $0.78 \mathrm{~m}^{3} / \mathrm{s}$, and the maximum discharge was $5.53 \mathrm{~m}^{3} / \mathrm{s}$. Based on these value boundaries, the scenarios corresponding with the discharge change were equally divided as relative levels of $1.5 \mathrm{~m}^{3} / \mathrm{s}, 3.0 \mathrm{~m}^{3} / \mathrm{s}$ and $4.5 \mathrm{~m}^{3} / \mathrm{s}$. Accordingly, the scenarios of discharge increase and decrease were showed in Figs. 8 and 9. Owing to the small headwork structure of the HorikawaYousui Irrigation Canal, the timing for the input discharge change was set at $5 \mathrm{~min}$. After $5 \mathrm{~min}$, the discharge remained at a constant value. Fig. 10 showed an example of the discharge increase scenarios where the discharge for the upstream boundary conditions increased from $0.78 \mathrm{~m}^{3} / \mathrm{s}$ to $5.53 \mathrm{~m}^{3} / \mathrm{s}$ within $5 \mathrm{~min}$ of opening the gate. The discharge gradually propagated from upstream to downstream and after around $1 \mathrm{~h}$, the expected discharge equaled $95 \%$ of the total discharge arriving downstream under the steady stage.

With regard to the Kim Son River, the scenarios of the arrival time of the discharge change propagation were performed in considering two options: (i) including diversion discharges and (ii) without diversion dis-

Table 2. RMSE indicators for the validation of the model in the Horikawa-Yousui Irrigation Canal

\begin{tabular}{cc}
$\begin{array}{c}\text { RMSE for the model using the observed discharge } \\
\text { and water level as boundary conditions } \\
(\mathrm{m})\end{array}$ & $\begin{array}{c}\text { RMSE for the model using the observed discharge } \\
\text { and the calculated water level by uniform flow } \\
\text { assumption as boundary conditions } \\
(\mathrm{m})\end{array}$ \\
\hline 0.136 & 0.196 \\
\hline
\end{tabular}

Table 3. RMSE indicators for the validation of the model using the observed discharge and water level as boundary conditions at the Bao Dap sluice gate in the Kim Son River

RMSE for the model using the observed discharge and water level as boundary conditions

(m)
RMSE for the model using the observed discharge and the calculated water level by uniform flow assumption as boundary conditions 
charge. There were five levels of discharge change as same as the scenarios level in the Horikawa-Yousui Irrigation Canal, which were also identified by the maximum and minimum discharges during the irrigation period, particularly from January to May owing to the flood season occurring from June to December. Different from the Horikawa-Yousui Irrigation Canal, the upstream discharge of Kim Son River was the discharge under the sluice gate and it was calculated according to the submerged overflow and complete overflow using the observed water levels upstream and downstream of the gate. Based on these conditions, the calculated minimum discharge during the irrigation period was $8.0 \mathrm{~m}^{3} / \mathrm{s}$ and the calculated maximum discharge was $40.0 \mathrm{~m}^{3} / \mathrm{s}$.

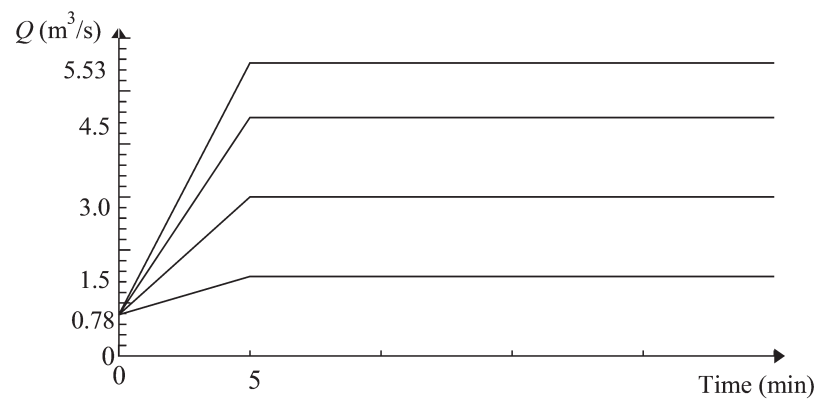

Fig. 8. Scenarios of discharge increase for the Horikawa-Yousui Irrigation Canal.

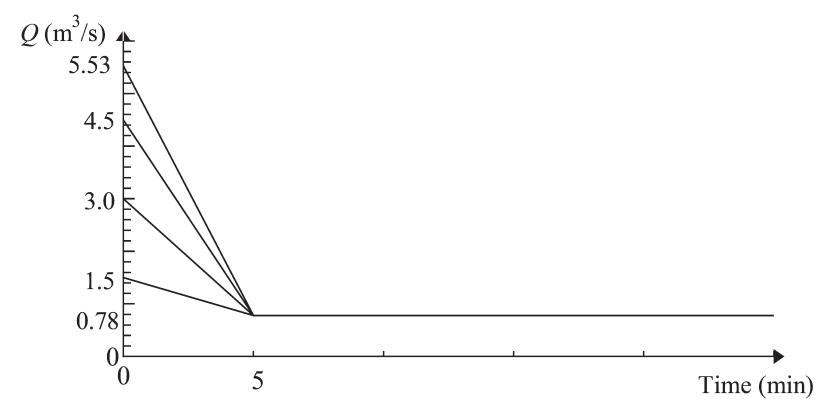

Fig. 9. Scenarios of discharge decrease for the Horikawa-Yousui Irrigation Canal.

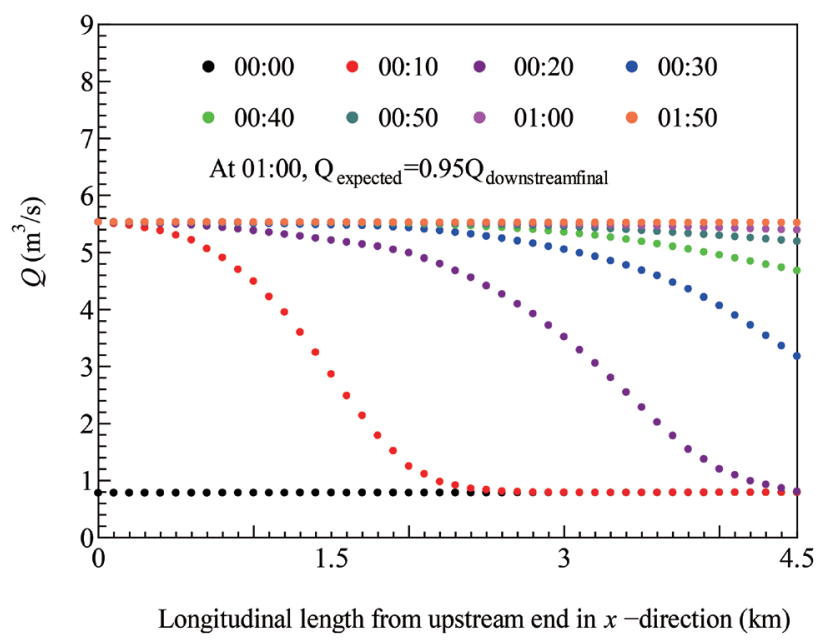

Fig. 10. Example of discharge increase scenarios in the HorikawaYousui Irrigation Canal case in consideration of no diversion discharge.
Based on these value boundaries, the scenarios corresponding with the discharge change were also equally divided as relative discharge levels of $16.0 \mathrm{~m}^{3} / \mathrm{s}$, $24.0 \mathrm{~m}^{3} / \mathrm{s}$, and $32.0 \mathrm{~m}^{3} / \mathrm{s}$. Correspondingly, the scenarios of discharge increase and decrease for the Kim Son River were indicated in Figs. 11 and 12, respectively. Differing from the Horikawa-Yousui Irrigation Canal, the timing for input discharge change was set to $30 \mathrm{~min}$ owing to its huge size and the number of gates at the Xuan Quan sluice gate (4 gates). After $30 \mathrm{~min}$, the discharge also remained constant. Fig. 13 presented an example of discharge increase scenarios wherein the discharge for upstream boundary conditions increased from $8.0 \mathrm{~m}^{3} / \mathrm{s}$ to $40.0 \mathrm{~m}^{3} / \mathrm{s}$ within $30 \mathrm{~min}$ of opening gate. The

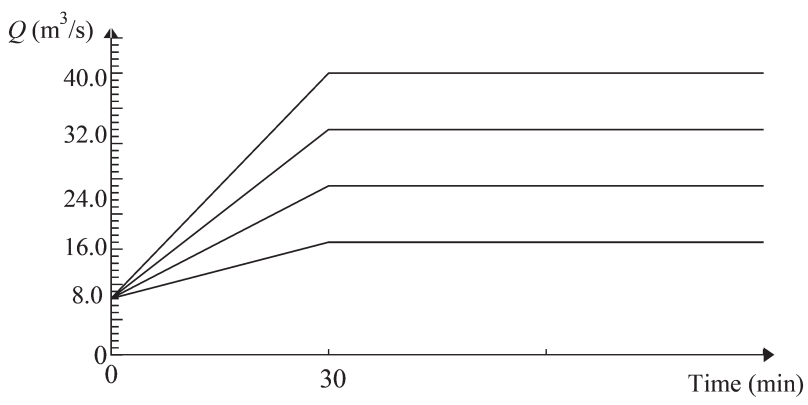

Fig. 11. Scenarios of discharge increase for the Kim Son River

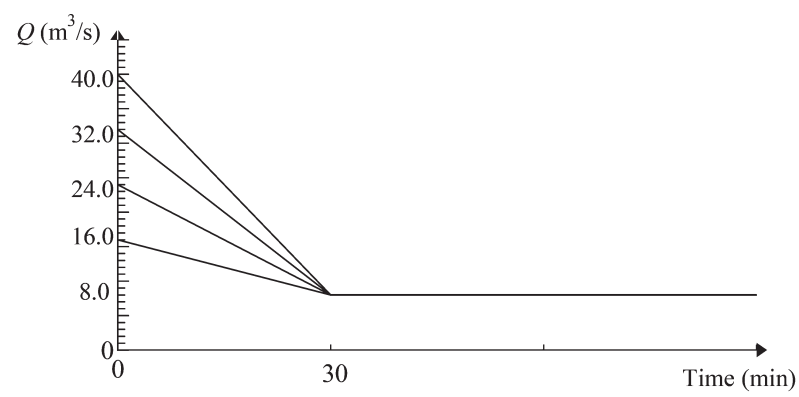

Fig. 12. Scenarios of discharge decrease for the Kim Son River.

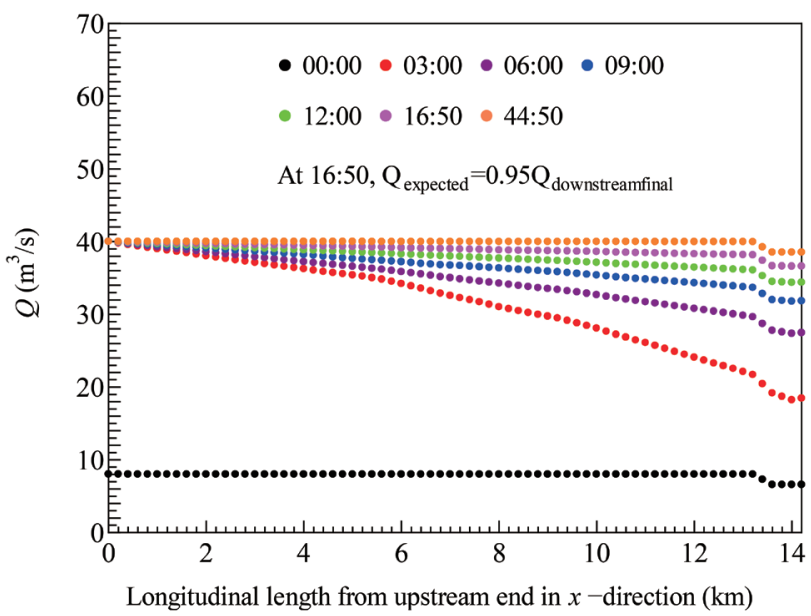

Fig. 13. Example of discharge increase scenarios in the Kim Son River case considering diversion discharges. 
discharge gradually propagated from upstream to downstream and after nearly $17 \mathrm{~h}$, the expected discharge was 95\% of total discharge arriving downstream under the steady stage. Besides, there was a slight drop in discharge near the downstream because a diversion discharge point appeared at this position.

Figs. 14 and 15 relatively illustrated the arrival time of increasing and decreasing discharge change propagation in minutes considering no diversion discharge for the Horikawa-Yousui Irrigation Canal. Accordingly, as can be clearly seen that in the case of increasing discharge from $0.78 \mathrm{~m}^{3} / \mathrm{s}$ in Fig. 8, a higher discharge increase led to a smaller arrival time of discharge change propagation from upstream to downstream. On the contrary, in the case of decreasing discharge, a smaller amount of discharge decreasing to $0.78 \mathrm{~m}^{3} / \mathrm{s}$ led to a smaller arrival time of discharge change propagation. Generally, in total for both of two cases, for the 4.5-km length of the Horikawa-Yousui Irrigation Cannal, the arrival time of discharge change propagation from upstream to downstream ranged from nearly $1 \mathrm{~h}$ to $1.5 \mathrm{~h}$.

The arrival time of increasing and decreasing discharge propagation from/to $8.0 \mathrm{~m}^{3} / \mathrm{s}$ in the case of including diversion discharges for the Kim Son River was shown in Figs. 16 and 17. On the contrary, for the Horikawa-Yousui Irrigation Canal, a higher discharge increase led to a higher arrival time of discharge change propagation. This was because a higher discharge increase led to a greater change in water level that required longer time for the propagation of flow downstream and reaching the steady stage. The total arrival times ranged from around $10 \mathrm{~h}$ to nearly $17 \mathrm{~h}$ for the 14.2-km length of the Kim Son River when discharge increased or decreased to four levels of discharge change. Figures 18 and 19 indicated the arrival time of

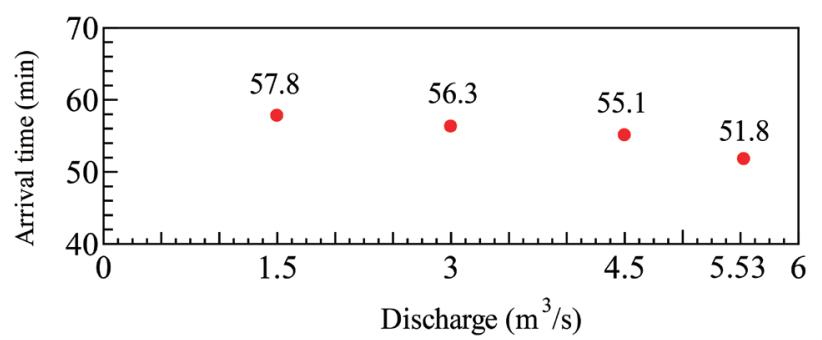

Fig. 14. Summary of arrival times of increasing discharge propagation from $0.78 \mathrm{~m}^{3} / \mathrm{s}$ in consideration of no diversion discharges for the Horikawa-Yousui Irrigation Canal.

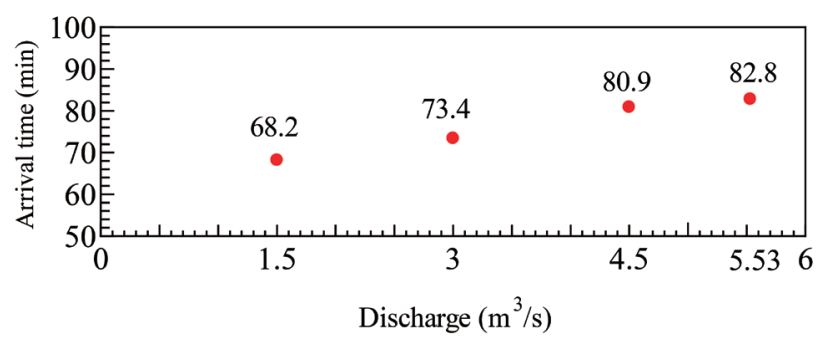

Fig. 15. Summary of arrival times of decreasing discharge propagation to $0.78 \mathrm{~m}^{3} / \mathrm{s}$ considering no diversion discharges for the Horikawa-Yousui Irrigation Canal. increasing and decreasing discharge propagation from/to $8.0 \mathrm{~m}^{3} / \mathrm{s}$ in consideration of no diversion discharge for the Kim Son River. Same phenomenon of the arrival time was observed in this case comparing with the case of including diversion discharges. Moreover, the total arrival time of discharge change in the case of no diversion discharge was a bit longer than the case of including diversion discharge.

On comparing the arrival time in the HorikawaYousui Irrigation Canal and Kim Son River, it can be

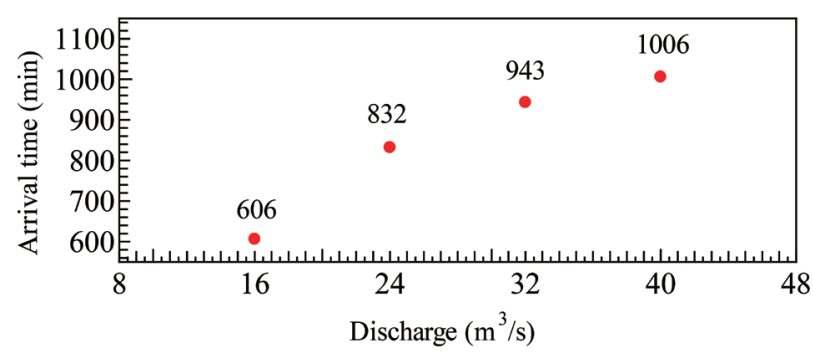

Fig. 16. Summary of arrival time of increasing discharge propagation from $8.0 \mathrm{~m}^{3} / \mathrm{s}$ considering diversion discharges for the Kim Son River.

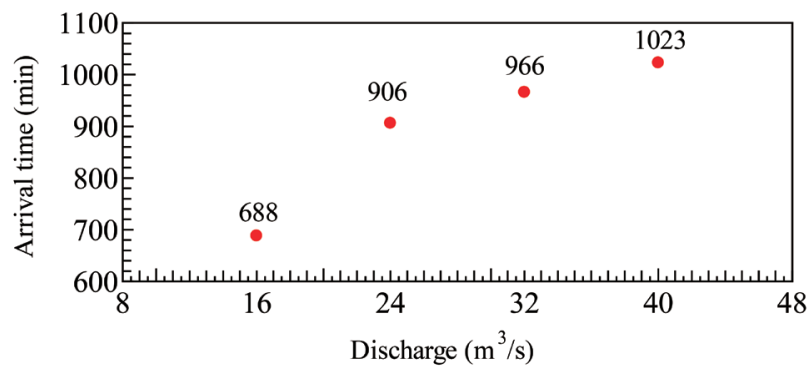

Fig. 17. Summary of arrival time of decreasing discharge propagation to $8.0 \mathrm{~m}^{3} / \mathrm{s}$ considering diversion discharges for the Kim Son River.

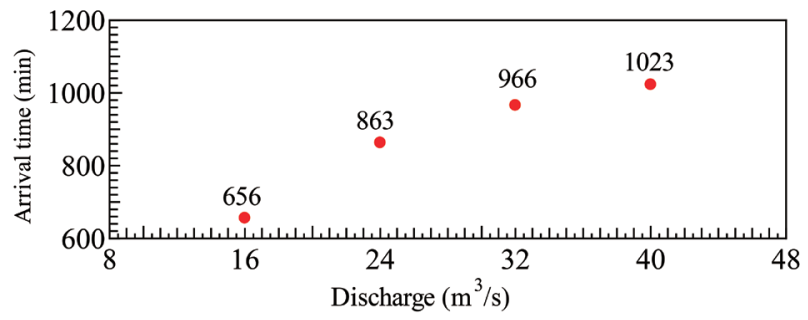

Fig. 18. Summary of arrival time of increasing discharge propagation from $8.0 \mathrm{~m}^{3} / \mathrm{s}$ considering no diversion discharge for the Kim Son River.

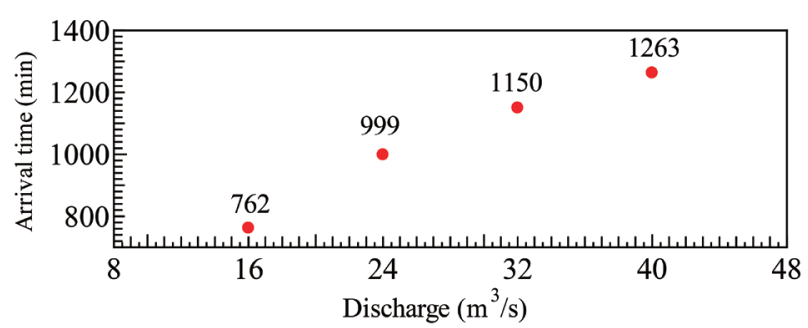

Fig. 19. Summary of arrival time of decreasing discharge propagation to $8.0 \mathrm{~m}^{3} / \mathrm{s}$ considering no diversion discharge for the Kim Son River. 
clearly seen that around three times increasing of length of canal was in regard to an approximate $10 \times$ increase in the arrival time of discharge change propagation. This was because the longer time of gate opening, which was 30 min in case of the Kim Son River compared with 5 min in case of the Horikawa-Yousui Irrigation Canal, caused the gradual change in water level and discharge that resulted in a longer change propagation. Besides, this phenomenon was the consequence of a sudden increase or decrease in the bottom elevation at some positions as well as nearly the horizontal bottom gradient along the Kim Son River, as shown in Fig. 3, leading to a greater arrival time of discharge propagation downstream and reaching the steady stage.

\section{Surge propagation}

A solitary wave is a type of surge/bore that is usually formed when there is a sudden discharge change in an open channel, and it propagates from upstream to downstream in a certain amount of time (Chow, 1959). The difference between the celerity of surge propagation from upstream to downstream and the average velocity calculated from the arrival time of increasing discharge change propagation was examined from the viewpoint of their similarity in the Horikawa-Yousui Irrigation Canal that is an artificial canal and had well-regulated discharge change propagation, compared to the Kim Son River.

The celerity $c(\mathrm{~m} / \mathrm{s})$ of surge propagation was calculated by the equation (Chow, 1959):

$$
c=\sqrt{\frac{g y_{2}}{2 y_{1}}\left(y_{1}+y_{2}\right)}
$$

where $y_{1}$ is the downstream water depth of the surge (m) and $y_{2}$ is the upstream water depth of the surge (m). Table 4 demonstrated the comparison between the calculated celerity for the surge propagation and the average velocity calculated from the arrival times of increasing discharge propagation in the Horikawa-Yousui Irrigation Canal. The celerity was approximately calculated using the average water depths along the entire length of the targeted canal at a nearly steady state immediately before changing discharge and after discharge change arrival. The celerity was nearly double in comparison with the average velocity. The difference between the calculated celerity and the average velocity was owing to the 5-min setting time of opening the gate that resulted in the gradual wave shape shown in Fig. 10 and the disappearance of the characteristics of surge propagation.

\section{CONCLUSIONS}

In terms of applying information communication technology in the operation of irrigation systems and utilizing smart irrigation, information regarding arrival time of discharge change propagation is indispensable to automatically monitor and operate structures and facilities along the river/canal and improve irrigation efficiency. In this study, we developed a one-dimensional gradually varied flow model for determining the arrival time of discharge change propagation from upstream to downstream. The targeted canal and river were the Horikawa-Yousui Irrigation Canal in Japan and the Kim Son River belonged to the Bac Hung Hai Irrigation System in Vietnam. The explicit finite difference method - Leapfrog scheme was used for a simplified computational procedure of solving the Saint-Venant equations. FORTRAN was utilized for reducing computational time.

Regarding the validation in the targeted canal and river, RMSE was used as an indicator to evaluate the differences between the simulated results by the model and observed data. The validation results indicated that it was in allowable range for RMSE and showed slight errors between the simulations and observed data.

In addition, the results of scenario analyses showed that the arrival time of discharge change propagation from upstream to downstream in case of the HorikawaYousui Irrigation Canal was around 1 to $1.5 \mathrm{~h}$, while it was approximately 10 to $17 \mathrm{~h}$ in case of the Kim Son River. Besides, in most of the cases, a normally higher discharge increase or decrease led to a higher arrival time of discharge change propagation from upstream to downstream. Moreover, the longer distance of the canal or river resulted in a longer arrival time for discharge propagation. Particularly, around a $3 \times$ increase in the canal length led to an approximately $10 \times$ increase in the arrival time of discharge change propagation. Basically, this was due to the longer time of gate opening significantly affecting the gradual change in water level and discharge that resulted in a longer propagation of change. Besides, this phenomenon consequently led to a sudden increase or decrease in the bottom elevation at some positions as well as a nearly horizontal bottom gradient along the Kim Son River. Moreover, the comparison between the celerity of surge propagation and the average velocity of discharge change propagation sug-

Table 4. Comparison between the calculated celerity for the surge propagation and the average velocity calculated from the arrival times of increasing discharge propagation from $0.78 \mathrm{~m}^{3} / \mathrm{s}$ in the case of the Horikawa-Yousui Irrigation Canal

\begin{tabular}{lcc}
\hline Scenarios of discharge change & Celerity $(\mathrm{m} / \mathrm{s})$ & Average velocity $(\mathrm{m} / \mathrm{s})$ \\
\hline 1. From $0.78 \mathrm{~m}^{3} / \mathrm{s}$ to $1.5 \mathrm{~m}^{3} / \mathrm{s}$ & 1.547 & 0.771 \\
2. From $0.78 \mathrm{~m}^{3} / \mathrm{s}$ to $3.0 \mathrm{~m}^{3} / \mathrm{s}$ & 1.430 & 0.751 \\
3. From $0.78 \mathrm{~m}^{3} / \mathrm{s}$ to $4.5 \mathrm{~m}^{3} / \mathrm{s}$ & 1.378 & 0.735 \\
4. From $0.78 \mathrm{~m}^{3} / \mathrm{s}$ to $5.53 \mathrm{~m}^{3} / \mathrm{s}$ & 1.355 & 0.691 \\
\hline
\end{tabular}


gested that the effects on these propagations are owing to the longer or shorter time of opening the gate led to a gradual or sudden change of discharge, respectively.

Based on the information regarding the accurate arrival time of discharge change propagation from upstream to downstream, farmers or irrigation workers can easily control and monitor appropriate discharge to save water as much as possible in terms of climate change and water scarcity in the near future. However, this one-dimensional gradually varied flow model was constructed for a single irrigation canal and natural river without consideration of complex systems. In a future study, we need to develop this model for a network system with nodes or a cycling route of channels.

\section{AUTHOR CONTRIBUTIONS}

M. Q. KHANH was mainly in charge of carrying out the research. K. HIRAMATSU helped in orientations, solutions, and conducted field surveys together with $\mathrm{M}$. Q. KHANH and T. TABATA. M. HARADA did research reviews and prepared the manuscript. All authors assisted in editing the manuscript and approved the final version.

\section{ACKNOWLEDGMENTS}

This study was mainly supported by the Japanese Grant Aid for Human Resource Development Scholarship (JDS) and partly by the JSPS Grant-in-Aid for Scientific Research (A) (Grant number 18H03968) for conducting field survey.

\section{REFERENCES}

Akgiray, O. 2005 Explicit solutions of the Manning equation for partially filled circular pipes. Canadian Journal of Civil Engineering, 32(3): 490-499

Chow, V. T. 1959 Open-Channel Hydraulics. McGraw-Hill Book Com (United States), pp. 98-123, 537-543, 554-557

FANG, H., CHEN, M. and CHEN, Q. 2008 One-dimensional numerical simulation of non-uniform sediment transport under unsteady flows. International Journal of Sediment Research, 23(4): 316-328

Ferreira, D. M., Fernandes, C. V. S. and Gomes, J. 2017 Verification of Saint-Venant equations solution based on the lax diffusive method for flow routing in natural channels. Brazilian Journal of Water Resources, 22(0): 1-10

Horvat, M., Horvat, Z. and Isic, B. 2017 Development, Calibration and Verification of a 1-D Flow Model for a Looped River Network. Environmental Modeling and Assessment, 22(1): 65-77

Jägermeyr, J., Gerten, D., Heinke, J., Schaphoff, S., Kummu, M. and Lucht, W. 2015 Water savings potentials of irrigation systems: global simulation of processes and linkages. Hydrology and Earth System Sciences, 19(7): 3073-3091

Kitamura, Y. 1990 Management of irrigation systems for rice double cropping culture in the tropical monsoon area. Technical Bulletin of the Tropical Agriculture Research Center, 27: 91-105

Meinzen-dick, R. 2014 Property rights and sustainable irrigation: A developing country perspective. Agricultural Water Management, 145: 23-31

Schaffranek, R. W. 1987 Flow model for open-channel reach or network. United States Geology Survey Professional Paper 1384, 7(3): 4-5 